\title{
WHAT IS AN ENGINEER: STUDY DESCRIPTION AND CODEBOOOK DEVELOPMENT
}

\author{
Sylvie Doré( ${ }^{(1)}$, Jillian Seniuk Cicek ${ }^{(2)}$, Marnie V. Jamieson ${ }^{(3)}$, Patrick Terriault ${ }^{(1)}$, Christian Belleau ${ }^{(1)}$, Renato \\ Bezerra Rodrigues ${ }^{(2)}$ \\ (1) Mechanical Engineering École de technologie supérieure (ÉTS), ${ }^{(2)}$ Centre for Engineering Professional Practice \\ and Engineering Education University of Manitoba,${ }^{(3)}$ Department of Chemical and Materials Engineering, \\ Faculty of Engineering, University of Alberta \\ sylvie.dore@etsmtl.ca, Jillian.SeniukCicek@umanitoba.ca, mvjamies@ualberta.ca
}

\begin{abstract}
There are still many questions to answer regarding the implementation and ongoing use of the $C E A B$ graduate attributes (GAs) to guide engineering education content and student progression. How well do our students know and understand the twelve GAs? Which ones do they find most important? Do the knowledge and importance of the GAs vary over the course of students' programs, or among institutions? Do students' definitions of an engineer reflect the GAs? How do the definitions reflect students' evolving understanding of engineering identity? Given the similarity of purpose, philosophy, and complementarity of questions and methods regarding the student perspective on engineering, the GAs, and their development, researchers from three institutions across Canada joined forces to conduct a national study. The overarching objectives of the study are to provide insight on how undergraduate students' engineering identities develop through the course of their programs using the $C E A B$ GAs as a normative framework, and enable meaningful comparisons of the GAs rankings and learning cultures at the three institutions. The objectives of this paper are to present an overview of the study development, and describe the methods used to develop the French and English codebooks for analysis of the qualitative data. We discuss the disambiguation of the codes and lexicon with particular attention to the concepts of professionalism and leadership and the emergence of three inductive codes: Engineering Work, Societal Improvement, and Personal Characteristics. We close the paper with a few words on future work.
\end{abstract}

Keywords: $\mathrm{CEAB}$ graduate attributes, undergraduate students, defining engineer, engineering identity, lexicon, codebook, inductive and deductive coding, disambiguation, bilingual

\section{INTRODUCTION}

Learning takes place as a result of what the student does [18]. The direction and content of student learning is guided by the program, course, and learning activity objectives and assessments [2,6]. Instructors are responsible for facilitating the learning process and managing the content, activities presented in the learning environment, and the learning culture in the courses they teach. Administrators are responsible for providing the infrastructure for the learning environment, managing the program and institutional learning culture, and building the program to provide a coherent pathway for student development. Students are responsible for participating in learning activities to construct their learning and make sense of the material. As stated by the theme of the 2020 Annual CEEA-ACEG conference, "To fully achieve their educational outcomes, students must be responsible for their own learning." We would argue that in order for students to be responsible for their own learning in engineering, they should be aware of the expected outcomes: Students should have an opportunity to develop an accurate perspective of what an engineer is and what an engineer does as they engage in their engineering education.

In engineering education, the graduate attributes (GAs), as outlined in the Washington accord, "are intended to assist Signatories and Provisional Members to develop outcomes-based accreditation criteria for use by their respective jurisdictions" [7]. As most engineering educators in Canada know, the Canadian Engineering Accreditation Board (CEAB) defines 12 graduate attributes that signify the outcomes graduates of all Canadian accredited engineering programs must possess. These outcomes guide the teaching/learning process throughout the curricula and are now part of the accreditation procedure. As such, all Canadian engineering students should be fully aware of the $12 \mathrm{GAs}$ in order to responsibly and effectively direct their learning.

\subsection{Motivation}

There are still many questions to answer regarding the implementation and ongoing use of the engineering GAs to guide engineering education and student progression. How well do our students know and understand these 12 GAs? Which ones do they find most important? Do the knowledge and importance of the GAs vary over the course of students' programs or across institutions? How 
do students' definitions of an engineer reflect their evolving understanding of engineering identity? How are the GAs reflected in these engineering identities, and what does this tell us about learning content, delivery, and culture across institutions?

The authors of this paper have a prior interest in this subject. At the University of Alberta (UofA), Jamieson and Shaw [11] investigated the learning theories that underlies the CEAB GAs and the learning environment or culture that may be required to effectively develop the GAs. They categorized the GAs using behaviorist (empiricist), cognitive (rationalist), and situative/ contextual (pragmatic/functionalism) tags, and studied the philosophical underpinnings. Based on further analysis of the $\mathrm{CEAB}$ GAs, a theoretical framework intended to support a learning culture for student GA development was proposed. The framework consists of four themes: core technical content, socio-contextual content, metacognitive skills, and professional skills. Tonso [19] states "...Identity matters. Engineering education provides a crucible for becoming engineers..." (244), clearly suggesting the guiding goals and learning culture of an engineering education program chart the course for the early development of an engineering identity. In addition, Jamieson and Shaw used an individual skill selfassessment as a pre-course team development and postcourse reflective activity in design courses $[9,10]$. The list of skills provided to students were developed using multiple design instructors' observations of the skills successful capstone design teams possessed and later classified according to the 12 CEAB GAs. These assessments enabled instructors to identify students' perspectives regarding their personal skill development through the lens of the GAs. In a separate arms-length closed-ended survey students were asked to evaluate their individual development of the GAs with similar results $[9,12]$.

Using a closed-ended rating survey, Seniuk Cicek et al. [14] determined the level of familiarity with, the relative importance of, and the levels of dependencies of the CEAB GAs as perceived across three groups of engineering stakeholders (students, faculty, and industry) in the Price Faculty of Engineering at the University of Manitoba (UofM). Participants were provided the GA $\mathrm{CEAB}$ definitions. Dependency findings were used to suggest a framework by which to view the GAs in groups of importance, interpreted using Bloom's taxonomy [16], and to determine the content validity of an engineering program based on the perceived relative importance of the GAs by stakeholders [15]. In contrast to Jamieson's theoretical study of the GAs with respect to philosophical and learning theory orientation [11], Seniuk Cicek et al. [14] engaged stakeholders with the definitions of the GAs to classify the GAs into four 'dependent' groups: problem solving skills, ethical reasoning, creativity and innovation, and interpersonal skills.
In 2019, Doré, Terriault, and Belleau [5] ran a pilot study in the Mechanical Engineering program at École de technologie supérieure (ÉTS) in which they asked mechanical engineering students to write a list of keywords pertaining to and a definition of an engineer. The collection of keywords and the definition provided by one student constitute a "definition set". They deductively coded the answers using the CEAB GA definitions with the intent of counting the number of GAs defined in each set and the frequency of the GAs across all sets. The underlying assumption was that the GAs are connected to what an engineer is and does, and therefore, the GAs would be identifiable in students' definitions of an engineer, and the frequency in which a GA appeared across the population would signify its importance. In contrast to Seniuk Cicek's study [15], participants were not given the list nor definitions of the GAs.

Table 1 displays the top ranked GAs by engineering students at the three institutions. The ranking reflects three different measures of importance: the most frequently included in students' definitions of an engineer (ÉTS); the most developed as assessed by students at the end of their capstone design course (UofA); and the most important as determined by students completing the frequency and criticality of use in engineering practice survey (UofM).

Table 1: Top ranked CEAB GAs across institutional student sample groups.

\begin{tabular}{|l|l|l|}
\multicolumn{1}{|c|}{$\begin{array}{c}\text { ÉTS } \\
{[\mathbf{5}]}\end{array}$} & \multicolumn{1}{|c|}{$\begin{array}{c}\text { U of Alberta* } \\
{[\mathbf{1 2}]}\end{array}$} & \multicolumn{1}{c|}{$\begin{array}{c}\text { U of Manitoba } \\
{[\mathbf{1 5}]}\end{array}$} \\
\hline Design & Design & $\begin{array}{l}\text { Individual and } \\
\text { team work }\end{array}$ \\
\hline Problem analysis & Problem analysis & Communication \\
\hline Knowledge base & Engineering tools & Professionalism \\
\hline $\begin{array}{l}\text { Economics and } \\
\text { Project } \\
\text { Management }\end{array}$ & $\begin{array}{l}\text { Individual and } \\
\text { team work }\end{array}$ & Ethics and Equity \\
\cline { 2 - 3 } & Communication & \\
\hline
\end{tabular}

* All five were equally ranked (agree/ strongly agree)

Though a direct comparison cannot be made as the study designs, objectives, and measures were different; it is of interest to consider students' perspectives on the GAs across three Canadian institutions in the current study.

\subsection{Study Objectives}

Given the similarity of purpose, philosophy, and complementarity of questions and methods regarding the student perspective on what an engineer is and does and the GAs, researchers from these three institutions joined forces to continue this work. The overarching objectives of the study are to provide insight on how undergraduate 
students' engineering identities develop through the course of their programs using the CEAB GAs as a normative framework, and enable meaningful comparisons of the GAs rankings and learning cultures across three institutions: the UofA, UofM, and ÉTS.

\subsection{Paper Objective and Structure}

The objectives of this paper are to present an overview of the study development, from origin to pilot to full study (Section 2. Background), and describe the methods used to develop the French and English codebooks for analysis of the qualitative data (Section 3. Codebook Development). We discuss the emergence of three inductive codes, and the disambiguation of the codes and lexicon (Section 4. Emergent Codes \& Disambiguation). We close the paper with a few words on future work.

\section{OVERVIEW}

This study has evolved through three phases, beginning with the origin studies - "What is an Engineer?" [5], "The relative importance of the GAs" [14-16]; and "Student pre and post course GA self assessment" [10] and "Defining an engineering learning culture" [11] - followed by a pilot study, run in 20192020, and a full study, which is currently being conducted. This trajectory is elucidated in Table 2, and the three phases of the study are discussed herein. (Note: only [5] is detailed here, as this is where the French codebook for the full study originated and this work focuses only on the definition of an engineer data sets collected. See $[8-12 ; 14-17]$ for a description of the other origin studies.)

\subsection{Origin Study}

Doré et al. [5] (Table 2, Origin Study) developed an assessment method to capture the perception of students for program accreditation purposes in the Mechanical Engineering program at ÉTS. It consisted of asking students to record a list of keywords they associate with being an engineer, and to write a formal definition of a (mechanical) engineer. The students did not have access to the list of GAs nor the CEAB GA definitions during this exercise. The raw data was closed coded in French using deductive coding for the 12 CEAB GAs as defined by Engineers Canada. As students did not always employ the same wording as presented in the CEAB definitions, it was necessary to build a French lexicon of synonyms for each GA through the deductive coding process to ensure consistent coding. First-year, mid-program and final-year students in mechanical engineering at ÉTS participated in the study. Results were compared for the different student populations and for changes in students' perceptions across the program. Among other things, results showed a significant increase from first-year to senior-year in the number of GAs included in students' definitions.
Furthermore, when looking at which GAs were most often included in students' definitions, Doré et al. noticed a statistically significant increase in the inclusion of GA8 (Professionalism), GA9 (Impact of engineering on Society and the Environment), and GA10 (Ethics and Equity) from first-year to senior-year students. These results showed that first year students had a sense of what an engineer does through the identification of the technical GAs, such as Design (GA4) and Problem Analysis (GA2). As students grew in seniority, they expressed more of what an engineer 'is' through the increased identification of these professional attributes (GAs 8, 9 and 10). The data offered a glimpse into how students' understanding of an engineer, or their engineering identity, shifts as students advance through their program.

\subsection{Pilot Study}

It is this observation of the shift in engineering identity at ÉTS that is the focus of the joint study (see Table 2, Pilot Study). The 2019-2020 academic year was devoted to obtaining ethics approval from all three institutions and entering into institutional data sharing agreements; refining the study methods (which included the data collection instrument and format; developing the demographic questions; defining the target populations); running a pilot study at ETS and UofM; and developing the lexicon for the English codebook.

For the pilot study, the questionnaire contained two parts. The first part reproduced the ÉTS qualitative assessment method developed in the origin study: asking students to record a list of keywords they associate with being an engineer, and write a formal definition of an engineer. The second part was a subset of Seniuk Cicek et al.'s quantitative closed-ended relative importance rating survey [14].

Three versions of the resulting questionnaires were produced: two in French, with one in paper and one in electronic format, and the other in English, in paper format. Data was collected at ÉTS in French and at the UofM in English. The surveys from ÉTS were used to compare the collection of qualitative data on paper and in electronic format. The data from the UofM was used to develop the English codebook. The development of the English codebook will be discussed in Section 3. The analysis of the data will not be presented in this paper.

\subsection{Full Study}

The full study (see Table 2) is being conducted presently. Both qualitative and quantitative data are being collected in the three participating institutions - ÉTS, UofM, and UofA. Because distance learning is the only teaching modality available for the 2020-2021 academic year due to the COVID-19 pandemic, an electronic questionnaire is being distributed via Google Forms to the 
Table 2. What is an Engineer: Study Trajectory

\begin{tabular}{|c|c|c|c|}
\hline Timeline & $\begin{array}{l}\text { Origin Study } \\
\text { (2016 to June 2019) }\end{array}$ & $\begin{array}{l}\text { Pilot Study } \\
\text { (June 2019-Sept 2020) }\end{array}$ & $\begin{array}{l}\text { Full Study } \\
\text { (Sept } 2020 \text { onward) }\end{array}$ \\
\hline \multirow[t]{3}{*}{ Projects } & $\begin{array}{l}\text { Doré et al. (2019) } \\
\text { What is an engineer? }\end{array}$ & \multirow{3}{*}{ Common pilot } & \multirow{3}{*}{ Common study } \\
\hline & $\begin{array}{l}\text { Seniuk Cicek (2018) } \\
\text { Determining the Relative } \\
\text { Importance of the CEAB Graduate } \\
\text { Attributes for Engineering: An } \\
\text { Exploratory Case Study }\end{array}$ & & \\
\hline & $\begin{array}{l}\text { Jamieson et al. (2019) } \\
\text { Defining an Engineering Learning } \\
\text { Culture; Pre-Post Course student } \\
\text { self assessment. (2016, 2017) }\end{array}$ & & \\
\hline Focus & $\begin{array}{l}\text { GA Development, Stakeholder } \\
\text { Importance, and Implementation }\end{array}$ & $\begin{array}{l}\text { Identity development } \\
\text { based on the GA }\end{array}$ & $\begin{array}{l}\text { Identity development } \\
\text { based on the GA }\end{array}$ \\
\hline Aim & $\begin{array}{l}\text { Understand aspects of the } \\
\text { Graduate Attributes of interest }\end{array}$ & $\begin{array}{l}\text { Refine methods and } \\
\text { leverage commonality }\end{array}$ & $\begin{array}{l}\text { Conduct comparative } \\
\text { research study }\end{array}$ \\
\hline $\begin{array}{l}\text { Data } \\
\text { collection }\end{array}$ & $\begin{array}{l}\text { ÉTS - Qualitative } \\
\text { UofM - Mixed Methods } \\
\text { UofA - Qualitative }\end{array}$ & $\begin{array}{l}\text { ÉTS \& UofM } \\
\text { Qualitative \& } \\
\text { Quantitative }\end{array}$ & $\begin{array}{l}\text { UofM, ÉTS and UofA } \\
\text { Qualitative \& } \\
\text { Quantitative }\end{array}$ \\
\hline Instruments & $\begin{array}{l}\text { Separate questionnaires at the } \\
\text { three institutions }\end{array}$ & $\begin{array}{l}\text { Common questionnaire } \\
\text { based on Doré et al. and } \\
\text { Seniuk Cicek et al. }\end{array}$ & $\begin{array}{l}\text { Common questionnaire } \\
+ \text { demographic } \\
\text { questions }\end{array}$ \\
\hline Format & $\begin{array}{l}\text { Paper data collection (ÉTS) } \\
\text { Paper \& electronic data collection } \\
\text { (UofM) } \\
\text { Theoretical work (UofA) } \\
\text { Paper and electronic data } \\
\text { collection for pre-post test (UofA) }\end{array}$ & $\begin{array}{l}\text { Paper \& electronic } \\
\text { (ETS) } \\
\text { Paper (UofM) }\end{array}$ & $\begin{array}{l}\text { Electronic (Pandemic) } \\
\text { UofM, ÉTS and UofA }\end{array}$ \\
\hline $\begin{array}{l}\text { Target } \\
\text { Population }\end{array}$ & First year, middle, last year (ÉTS) & $\begin{array}{l}\text { First year, mid } \\
\text { program, last year } \\
\text { (ETS) } \\
\text { First / last year (UofM) }\end{array}$ & $\begin{array}{l}\text { First year, midprogram, } \\
\text { last year (ETS) } \\
\text { First \& last year (UofM } \\
\text { \& UofA) }\end{array}$ \\
\hline Codebook & $\begin{array}{l}\text { Phase } 1 \text { - Training \& Codebook } \\
\text { Development- French, } \\
\text { Deductive GA based Closed } \\
\text { Coding Rule Development }\end{array}$ & $\begin{array}{l}\text { Phase } 2 \text { - Training and } \\
\text { Analysis - Iterative } \\
\text { development of English } \\
\text { Deductive/Inductive } \\
\text { codebook with codes } \\
\text { allowed outside of GA }\end{array}$ & $\begin{array}{l}\text { Phase } 2 \text { - training } \\
\text { coders and possible use } \\
\text { of NVivo to code the } \\
\text { electronic data }\end{array}$ \\
\hline
\end{tabular}

student target populations. Aside from the online collection format, the only difference between the full study questionnaire and the one used in the pilot study is the addition of a number of demographic questions.

\section{CODEBOOK DEVELOPMENT}

We define codebook as a collection of words or expressions attributed to a particular set of codes. In our case, the deductive codes (i.e., the codes that existed before we analyzed the data) are the 12 CEAB GAs. The words and phrases we attribute to each code (GA) are the codebook lexicon, and they were derived from the data (i.e., they are based on the students' words/phrases that we coded for a specific GA). Our final codebook also contains three inductive codes that emerged in the data that were not captured by the $12 \mathrm{CEAB}$ GA codes, and three rules for when coding is not straightforward. Developing the codebook was a two-phase process.

\subsection{Phase 1 French Codebook}

As the study conducted at ÉTS in 2018-2019 was for accreditation purposes only, a deductive coding strategy was employed, with the 12 GAs forming the codes. A French codebook was developed. The codebook was constructed during a training session involving three francophone coders, all professors in mechanical engineering (one being the first author). They began the training session with a subset of 15 out of the 500 definition sets collected, and ended up coding 30 sets to 
reach saturation. The process was as follows: coders sat together and individually read and coded one set, and then confirmed or discussed that set until they reached consensus. They then moved to the next net, repeating this process, moving through 30 sets in this way until their codebook was developed. Discussions among coders usually resulted in a new word or phrase (i.e., lexicon) being added to the codebook for the agreed upon code. However, if disagreement persisted, a rule was formulated to define how to proceed in coding. Three rules were thus developed:

1. When in doubt, do not code. Some definitions were poorly formulated and the meaning of some words was impossible to decipher. When this occurred, these words were not coded. It was also determined that it is important not to overly interpret what students wrote. When there were arguments over interpretation, these words were not coded.

2. If the context is not clear, do not code. At times, the context for the code had to be determined. For example, the word 'responsible' can mean accountability (one accepts the consequences of their decisions or actions), which would be coded as GA8 (Professionalism), or being in charge (one is responsible for a task), which would be coded as GA11 (Economics \& project management), or as leadership, GA6 (Individual and teamwork). When coding, the CEAB GA definition was often consulted to uncover the intended context. When in doubt, Rule 1 was applied and the word/phrase was not coded.

3. In a given definition set, count a given code only once. Often a given code (GA) appeared multiple times within a definition set. As the objective was to identify if a given code was present rather than how often, we determined to count each code only once per student. Therefore, when we write that a student's definition set contains $\mathrm{X}$ number of codes, we mean that we encountered $\mathrm{X}$ number of different codes (i.e., different GAs).

The resulting codebook, reproduced in Annex I of Doré et al. [5] consisted of 25 words (i.e., lexicons) relating to the 12 codes (i.e., the 12 CEAB GAs). Certain GAs did not have any synonyms (additional lexicon), because the coders felt the CEAB definition was sufficiently clear. For example, this was the case for GA1- Knowledge base for engineering. Whenever the word knowledge or a specific subject matter appeared in the definition (e.g. mathematics, statics, fluid mechanics...), it was coded as GA1. GA5 - Engineering tools, GA6 - Individual and Teamwork and GA12 - Lifelong learning, completed the list of codes without lexicon.

\subsection{Phase 2 English Codebook}

The second phase was conducted during the pilot study during the 2019-2020 academic year, and started by translating the French codebook to English. Rich discussions regarding the English and French versions of the GAs and their eventual reconciliation in the codebook led to revisions in both codebooks. Following this was the training session for coding. The training sessions were conducted with a subset of 20 definition sets out the 341 collected at UofM during the 2019-2020 academic year. (Note: Twenty sets were chosen as 15 proved too little for the French codebook. For the English codebook, 20 sets enabled saturation). The first author first trained the second and third authors, who in turn trained a $\mathrm{PhD}$ student from UofM. The development of the phase 2 codebook was very much an iterative process, as each training session was followed by a series of discussions to reach consensus and add to the lexicon, with decisions supported by our diverse professional expertise and experiences, and the research literature. The codebook was enriched as the new coders noted nuances and novel items. This led to inductive coding of the data and the emergence of three new codes: Code13 - Engineering work (e.g. build, maintain), Code14 - Societal Improvement Activities, and Code15 - Personal Characteristics (discussed in detail in Section 4.1.)

After the Phase 2 codebook was developed the 341 UofM definition sets were coded. The sets were assigned a random number from 1 to 400 and placed in ascending numerical order to shuffle and track the data. Each set was coded twice, once by two different coders. Furthermore, all four coders were paired once with every other coder as shown in Table 3.

Coder pairs met to discuss their shared definition sets. When consensus could not be reached, or coder pairs wished to verify decisions, all four coders met. At this point, only minor adjustments were required for Codes 1 to 14. However, Code15- Personal characteristics, was greatly enriched (i.e., we developed a long list of lexicons) during this exercise.

The final codebook consists of over 200 words or expressions, with just over 100 words in Code15, and all codes are populated with lexicon. The three rules developed during Phase 1 were carried over to the Phase 2 codebook, and several disambiguation notes were made during the development of the Phase 2 codebook. During Phase 2 the French codebook was revised to maintain consistency for Phase 3.

Table 3. Student definition set coding overlap among the four coders (400 documents)

\begin{tabular}{|c|c|c|c|c|}
\hline Coder & A & B & C & D \\
\hline A & & $1-67$ & $68-134$ & $135-200$ \\
\hline B & $1-67$ & & $201-267$ & $268-334$ \\
\hline C & $68-134$ & $201-267$ & & $335-400$ \\
\hline D & $135-200$ & $268-334$ & $335-400$ & \\
\hline
\end{tabular}




\section{EMERGENT CODES \& DISAMBIGUATION}

Here we discuss the emergence of three inductive codes, and our process to disambiguate the GA codes.

\subsection{Emergence of codes}

The three inductive codes that emerged during the UofM definition set analysis are: Code13 - Engineering Work, Code14 - Societal Improvement Activities, and Code15 - Personal Characteristics.

Code13-Engineering Work. Students answering the question, What is an engineer? and supplying a definition of an engineer often used engineering work descriptions. One could argue that some engineering work is included in the GAs, such as GA2-Problem analysis, GA3Investigation, GA4-Design, as well as portions of GA9Impact of engineering on society and the environment and GA11-Economics and Project management. However, students included other engineering work in their definitions that described implementation, and hands-on tasks not found in the GAs. Therefore, a code for engineering work was added (Code13), and lexicons such as build, maintain, fabricate, were ascribed to this code.

Code14-Societal Improvement. GA8-Professionalism, describes the role of an engineer as the "protection of the public and the public interest" Which we characterize as non-malevolence (Do no harm). GA10-Ethics and Equity describes the standards and accountability of the behaviour of an engineer as they carry out their role in society. When students expressed the purpose of an engineer as 'improving society', 'making life better', they are referring to beneficence (Do good) that is neither present in GA8 nor GA10. To capture this difference in perspective, Code14 was named.

Code15-Personal Characteristics. Students often included personal characteristics in their definition of an engineer such as 'intelligent', 'organized', 'reliable', 'visionary', etc. Some of these epithets could be construed as extensions of one or another of the GAs. For example, one could be tempted to code 'intelligent' as GA1Knowledge base as intelligence is often connected to/implied by knowledge, or 'organized' as GA11Economics and Project management since project management is a specific organizational system and project managers plan and organize. However, if the context was not clear, we then found ourselves interpreting, which was against our coding rules. So many of these words emerged in the data that we decided to code them, as they were rich data for our exploration of engineering identity. Therefore, when the data in question is a general characteristic, it is coded as Code15. Thus, the descriptor organized is coded as Code15, but the verb to organize is coded as GA11.

\subsection{Disambiguation effort}

As discussed, the Phase 2 codebook had a more extensive lexicon than the Phase 1 codebook. This was due to the team's effort to disambiguate the words that had led to the formulation of Rules 1 and 2 . Disambiguation was supported by the team members' diverse professional backgrounds, diverse research studies on the GAs, our convictions, and the time we spent arguing our views. This led to rich discussions and a deeper understanding of the GAs, and an expanded lexicon. Of particular note are our discussion about GA8 and GA9; GA8 and GA10; the word leadership; and other contentious words.

GA8 and GA10. The Merriam-Webster dictionary defines professionalism as the 'conduct, aims, or qualities that characterize or mark a profession', which in turn is a 'calling requiring specialized knowledge' (referred to in GA1) 'and often long and intensive academic preparation.'

One of the definitions found in the same dictionary for ethics is 'the principles of conduct governing an individual or a group' and professional ethics is given as an example. Thus, we are to understand that the professionalism of an engineer is encapsulated in the engineering professional code of ethics: The two concepts are intimately linked making their differentiation difficult, which was demonstrated when we were coding students' definitions of engineer.

Ethics, as a discipline, also called moral philosophy, is concerned "with what is morally good and bad and morally right and wrong' as stated in the Britannica Encyclopedia. There exists many approaches to ethics, one of which is the duty-based, or deontological, approach. Kant argues that 'doing what is right is not about the consequences of our actions... but about having the proper intention in performing the action. The ethical action is one taken from duty, that is, it is done precisely because it is our obligation to perform the action' [3].

It is interesting to note that members of the Canadian Engineering Qualifications Board Practice Committee [4] define ethics as 'the discipline or field of study dealing with moral duty or obligation. This typically gives rise to a set of governing principles or values which in turn are used to judge the appropriateness of particular conduct or behaviour.' Although not explicitly stated, this definition leads us to see that the professional code of ethics for engineers is formulated in the tradition of the duty-based approach. To further this argument, it is noteworthy to mention that GA10 in French is named 'code de deontologie'.

Thereby, accountability is intertwined with duty, as it is viewed as an obligation or willingness to accept responsibility in GA8 - Professionalism, and to account for one's actions in GA10 - Ethics \& Equity. To disambiguate these concepts, we turned to the $\mathrm{CEAB}$ 
definitions. Whenever a word or expression in a student definition matched 'professional', 'protection of the public' etc., it was coded as GA8. If the definition referred to 'ethics', 'ethical', or universal values such as 'honesty', 'accountability', it was coded as GA10.

GA8-Professionalism and GA9-Impact of engineering on society are closely related inasmuch as GA9 describes how an engineer will carry out the role or purpose described in GA8. The differences between GAs 8 and 9 are reflected in the coding of the student responses. Data describing the role or purpose of an engineer, including data referencing 'protecting the public'; 'for the purpose of public safety', etc. are coded as GA8. Items that reference how engineers develop or evaluate sustainable solutions and/or designs including items such as 'with the effect of'; 'solves world's problems'; 'meets the needs of society by'; 'designing by the society's needs', etc. were coded as GA9, as evidence of analysis was apparent. Whatever the activity (design, maintain, build...), GA9 is not about creating the impact (GAs 2, 4, 8 or 14) but in analysing the effect of the activity with the primary objective of minimizing the negative impact of the activity in accordance with GA8 an engineers' professional obligation to protect the public and the public interest.

'Leadership'. During Phase 2, a new outlook was given to the word, leadership. All forms of leadership, including positional leadership, which had previously been considered a managerial task (GA11 - Economics and project management), were determined to all under GA6 - Individual and Teamwork. This was because we determined that management is a business practice involving activities such as planning, controlling, coordinating, allocating resources, scheduling, and managing the plan [13]. Leadership is a skillset used in engineering and management involving activities such as developing a vision, motivating, leading, inspiring, creating alignment, directing and executing the plan $[13,8]$. However, the word, leader is explicitly defined in GA6. Therefore, we added leadership to the GA6 lexicon.

Other contentious words. At times, students wrote words such as 'constraints' and 'criteria' in their definition of engineer. We discussed whether these words reflected the 'specialized engineering knowledge appropriate to the program' described in GA1 Knowledge base, or were a part of 'an ability to design solutions for complex, open-ended engineering problems' as described in GA4 - Design. In this case, a determination was made to code this as the design (GA4) rather than Knowledge base (GA1) as constraints and criteria are omnipresent and inherent to design.

We had a similar tension for the word, synthesis. Synthesis is a cognitive activity that engineers use when carrying out the tasks of problem analysis, investigation and design; it could be used in GA7 - Communication Skills. Yet synthesis is only explicitly mentioned in Investigation (GA2). In this case, a determination could not be made for synthesis, and unless the context is clear in the data, Rule 1 is applied (do not code).

\section{CONCLUSIONS AND NEXT STEPS}

Three researchers from ÉTS, UofM, and UofA joined forces to conduct a study to learn how undergraduate students' engineering identities develop through the course of their programs. Using the CEAB GAs as a normative framework, the researchers will be able to make meaningful comparisons of the GAs rankings and learning cultures across these institutions. The objectives of this paper were to present an overview of the study development, describe the methods used to develop the French and English codebooks for analysis of the qualitative data, and discuss the disambiguation of the codes and lexicon. There was a shift of focus between the origin study conducted at ÉTS in 2018-2019, which led to the development of the Phase 1 French codebook, and the pilot study in 2019-2020, which led to the development of the Phase 2 English codebook. The origin study had the sole purpose of gathering GA data for accreditation, while the pilot and full studies are purposed to explore the development of students' engineering identity through their undergraduate programs using the GAs as the normative framework. This change in focus resulted in a change of coding strategy, which progressed from a deductive approach in Phase 1 to a deductive/inductive approach in Phase 2. As a result, three inductive codes emerged from the development of the English codebook, which give insight into students' understanding of engineer, and their engineer identity.

Currently, data are being collected for the full study at the three participating institutions. Qualitative and quantitative data from both the pilot study and the full study will be reported on soon. Overall, the iterative process demonstrated in both the development of this study and the phased development of the codebooks, supported by our diverse professional expertise and experiences, have led to an enriched understanding of the CEAB GAs and how students define engineer. We look forward to continuing this work and sharing our findings with the engineering education community.

\section{Acknowledgements}

Ethics approval for research involving humans has been granted at the three participating institutions for this study. Additionally, the University of Manitoba Office of Institutional Analysis has approved this research. We thank the CEEA-ACEG reviewers for their insights which will be helpful moving forward with the study. 


\section{References}

[1] Joanna Allan, "Learning outcomes in higher education", Studies in Higher Education, 21:1,93-108, 1996, DOI: $10.1080 / 03075079612331381487$

[2] John Biggs, "What the student does: teaching for enhanced learning", Higher Education and Development 18(1):57-75, 1999.

[3] Sheila Bonde and Paul Firenze. Making Choices: A Framework for Making Ethical Decisions. Providence, RI: Brown University, 11 pp., 2013. Available as of February 172021 from https://www.brown.edu/academics/science-and-technologystudies/framework-making-ethical-decisions

[4] Canadian Engineering Qualifications Board Practice Committee. Guideline on the Code of Ethics. Ottawa: Engineers Canada, 9 pp., 2012. Available as of February $17 \quad 2021$ from https://engineerscanada.ca/sites/default/files/guideline_code_w ith 1.pdf

[5] Sylvie Doré, Patrick Terriault, and Christian Belleau, "Gathering the Voice of the Students for Accreditation Purposes through their Definition of "Engineer." Proceedings of the Canadian Engineering Education Association Conference (CEEA/ACEG, Ottawa, ON, June 8-12). 8 pp., 2019.

[6] John Hattie, "The Black Box of Tertiary Assessment: An Impending Revolution". in L. H. Meyer, S. Davidson, H. Anderson, R. Fletcher, P.M. Johnston, \& M. Rees (Eds.), Tertiary Assessment \& Higher Education Student Outcomes: Policy, Practice \& Research (pp.259-275). Wellington, New Zealand: Ako Aotearoa, 2009.

[7] International Engineering Alliance (IEA), Graduate Attributes and Professional Competencies, 2013. IEA. http://www.ieagreements.org/

[8] Marnie V. Jamieson and John R. Donald "Building the Engineering Mindset: Developing Leadership and Management Competencies in the Engineering Curriculum" in Proc. (CEEA/ ACEG20), Montreal, QC, June 18-21, 8 pp., 2020

[9] Marnie V. Jamieson, "Application of blended and active learning to chemical engineering design instruction", University of Alberta, 2016.

[10] Marnie V. Jamieson and John M. Shaw, "Pre and Post Course Student Self-Assessment of CEAB Graduate Attributes - A Tool for Outcomes Assessment, Student Skill and Course Improvement", in Proc. (CEEA/ ACEG16), Dalhousie, NS, June 19-22), 8 pp., 2016.

[11] Marnie V. Jamieson and John M. Shaw, " Learning to Learn: Defining an Engineering Learning Culture" Proceedings of the Canadian Engineering Education Association Conference: (CEEA/ACEG, Ottawa, ON, June 8-12), 9pp., 2019

[12] Marnie V. Jamieson and John M. Shaw, "Student and Instructor Satisfaction and Engagement with Blended Learning in Chemical Engineering Design", in Proc. (CEEA/ACEG17) Paper 040; Toronto, ON, 2017
[13] William Schell, Bryce Hughes, John Donald, Tom Goldfinch, Anthony Kadi, Emily Moore, Doug Reeve, Cindy Rottmann, Patricia Sheridan, "Leadership Transcending Borders: Building Bridges to Integrate Technical and Professional Knowledge." In Proc. (CEEA/ACEG20, Montreal, QC, June 18-21), 8 pp., 2020

[14] Jillian Seniuk Cicek, Sandra Ingram, Danny Mann, and Robert Renaud. "Investigating the Relative Importance of the CEAB Graduate Attributes: Study Design and Initial Findings." Proceedings of the Canadian Engineering Education Association Conference: Innovation and Diversity in Engineering Education (CEEA/ACEG, Toronto, ON, June 4 7), 9 pp., 2017.

[15] Jillian Seniuk Cicek, (2018) Determining the Relative Importance of the CEAB Graduate Attributes for Engineering. An Exploratory Case Study at the University of Manitoba. $\mathrm{Ph}$.D. thesis, University of Manitoba. 368 pages, 2018.

[16] Jillian Seniuk Cicek and Robert Renaud. "Determining the Dependencies of Engineering Competencies for Engineering." Proceedings of the American Society for Engineering Education Conference: Charged Up for the Next 125 Years (ASEE, Tampa Bay, FL, June 16-19), 19 pp., 2019.

[17] Jillian Seniuk Cicek, Robert Renaud, Danny Mann, and Sandra Ingram. "Determining the Content Validity of a Biosystems Engineering Program." Proceedings of the Canadian Engineering Education Association Conference: Building Foundations for Student Success (CEEA/ACEG, Vancouver, BC, June 3-6), 8 pp., 2018.

[18] Herbert Simon, "What we know about learning". Journal of Engineering Education, (October) 1998.

[19] Karen L. Tonso, "Engineering identity.” In A. Johri, \& B. M. Olds (Eds.) Cambridge handbook of engineering education research. (pp. 267-282) New York, NY: Cambridge University Press, 2014 